\title{
Clinical spectrum of callosum corpus splenium lesions: subdiagnostics of a common entity
}

\begin{abstract}
The corpus callosum is the largest white matter commissure of the brain that communicates both cerebral hemispheres; and can be altered by multiples etiologies; some of them, with a pathophysiological mechanism not elucidated. There are some reports, about splenium callosum lesions, associated to nonspecific clinical condition, but with a common characteristic: disturbance of consciousness and wakefulness as the predominant symptom; although, also have been described seizures, headache or athetosis in some reports. Independent of previous features, this type of lesions, may be evidenced in asymptomatic patients, as a radiological finding. We present three cases of patients with these radiological features, in two of them which a common finding, was a uremic syndrome that does not required chronic dialisys. We concluded that in patients with transient impairment of consciousness of unclear origin, a callosum splenium lesion would to be suspect, especially if they are associated to uremic syndrome. Also, that these types of findings could be more frequent than expected, so more studies are necessary to clarify the physiopathology.
\end{abstract}

Keywords: callosum corpus lesions, consciousness disturbance, splenium callosum lesions, mild encephalitis/encephalopathy
Case Series

Volume 8 Issue 2 - 2018

\author{
Alberto Vargas Cañas,' Rodrigo Guerrero \\ Torrealba, ${ }^{2}$ Nicolas Rebolledo Villar ${ }^{3}$ \\ 'Unidad de Neurología, Hospital Santiago Oriente "Dr. Luis \\ Tisné B.”; Investigador Internacional Universidad Autónoma del \\ Caribe, Chile \\ 2Unidad de Neurología, Hospital Santiago Oriente "Dr. Luis \\ Tisné B.”, Chile \\ ${ }^{3}$ Servicio de Urgencias, Hospital Santiago Oriente “Dr. Luis Tisné \\ B.", Chile
}

Correspondence: Alberto Vargas Cañas. Unidad de Neurología, Hospital Santiago Oriente "Dr. Luis Tisné B.", Av. Las Torres 5100; Penalolen; Santiago de Chile, Chile, Tel +56224725513,Email amvc_md@yahoo.com

Received: October 26, 2017 | Published: March 06, 2018
Abbreviations: CC, callosum corpus; MERS, mild encephalitis/encephalopathy with a reversible isolated splenium callosum corpus lesion; LHECC, hypodense lesion in the splenius of the corpus callosum; BBB, blood-brain barrier; NU, neurology's unit; ED, emergency department; CT, computer tomography; MRI, magnetic resonance imaging; HD, hemodialysis ; ECG, electrocardiogram; ECHOC, echocardiogram; LP, lumbar punction; EEG, electroencephalogram

\section{Introduction}

The callosum corpus (CC) is the largest white matter commissure of the brain that communicates both hemispheres. ${ }^{1}$ Anatomically, can be divided in 3 portions: anterior, middle and posterior; two first that depends of anterior circulation; and the splenius of the corpus callosum of posterior respectively. ${ }^{1}$

The most frequent causes of injury of $\mathrm{CC}$ are cerebrovascular stroke, cranial brain trauma, central nervous system infections, alcohol, epilepsy, multiple sclerosis and MERS syndrome (mild encephalitis/ encephalopathy with a reversible isolated splenium callosum corpus lesion). ${ }^{2,3}$ Pekala et al. ${ }^{4}$ suggest to include patients who are or have undergone oncologic radiotherapy of the head and neck. ${ }^{4}$

The MERS's clinic is variable. Confusion or alteration of consciousness is the most frequent clinical sign. ${ }^{2}$ Another study considers fever and headache as a predominant symptomatology. ${ }^{3}$ Both mention dysarthria, seizures, hemiparesis and associated ataxias. And recently, athetosis was described by one of the authors. ${ }^{5}$

It's important to consider that in elderly patients, it is possible to find a silent hypodense lesion in the splenius of the corpus callosum (LHECC) as radiological findings; as well it has been mentioned in children; probably directly related to the inmaturity and involution of the central nervous system related to a fragility of the bloodbrain barrier $(\mathrm{BBB}){ }^{4}$ We communicate 3 patients hospitalized in our Neurology Unit (NU) between July and October 2017, due to confusional syndrome in two of them, and seizures in the remainder; and in which, neuroimaging studies showed a unique hypodensity in the splenius of the corpus callosum.

\section{Case presentation}

\section{Case I}

Male, of 65 years old, with diabetes mellitus, and hypertension both with poor treatment; suspended smoking and occasional alcohol consumption who has a emergency department (ED) consultation by 10 days of fluctuating confusional syndrome associated with headache, psychomotor agitation and gait instability. Is initially evaluated in the ED, with blood pressure level (231/122 mmhg), hemoglucotest (448 mg/dl); and physical examination that evidenced a arousal patient, time and place disorientated; and without focal neurological deficits. Laboratory tests showed a serum creatinine level in 3.29 , blood urea nitrogen 44 , glycemia $393, \mathrm{Ph} 7.33, \mathrm{HCO}_{3}$ 19 , Na 142, K 4.3. Computer tomography (CT) was informed as a microangiopathy of the supratentorial white matter and a left thalamic sequela stroke. In the NU, the patient evolves with more disturbance of consciousness and acute renal failure that required hemodialysis (HD) and evolve with a septicemia of pulmonary origin. Studies were complemented with echocardiogram (ECHOC), lumbar puncture (LP) and electroencephalogram (EEG); all they normal. Because the persistence of the symptoms, new CT shows a hypodensity in the splenius of the corpus callosum, and in a second look this finding was present in the first scanner (Figure 1) (Table 1); and a diagnosis of MERS syndrome was made.

\section{Case 2}

A 60 -year-old woman with diabetes mellitus type 2, hypertension, chronic obstructive pulmonary disease, and suspended smoking habit; with the previous antecedent of a recent hospitalization for 
digestive tract hemorrhage, and paroxystic atrial fibrillation in oral anticoagulant treatment with warfarin. One month later of this previous clinical antecedent, the patients come to ED by focal seizures auto-limited, without loss of consciousness; which was followed by left face arm paresis that reverted after hours. A Todd's phenomenon was diagnosed in ED. EEG normal, laboratory tests on the ED show

Table I Our clinical cases, co morbidities and relevant data

\begin{tabular}{|c|c|c|c|c|c|c|}
\hline Case & Sex/Age & Comorbidities & Symptomatology & $\begin{array}{l}\text { Neurological } \\
\text { examination in El) }\end{array}$ & Exams (highlight) & Outcome \\
\hline \multirow[t]{8}{*}{1} & 65 - Male & $\begin{array}{l}\text { Diabetes Mellitus } \\
\text { Hypertension }\end{array}$ & $\begin{array}{l}\text { Consciousness } \\
\text { disturbance }\end{array}$ & $\begin{array}{l}\text { High Stood Pressure } \\
231 / 122 \mathrm{~m} \mathrm{mhg}\end{array}$ & Cr 2.9 mgid I & Acute renal failure \\
\hline & & & Headache & $\begin{array}{l}\text { Capillary glycemic } 448 \\
\mathrm{mg} / \mathrm{d} 1\end{array}$ & BUN 44 mg/dl & Pulmonary Infection \\
\hline & & Smoking & & Disorientated & Glycemic serum & \\
\hline & & & & $\begin{array}{l}\text { Neurologic Deficit } \\
\text { described (-) }\end{array}$ & Electrolytes (-) & \\
\hline & & & & & EEG(-) & \\
\hline & & & & & ECHOC (-) & \\
\hline & & & & & EEG (-) & \\
\hline & & & & & LP(-) & \\
\hline \multirow[t]{9}{*}{2} & 60 - Female & Diabetes Mellitus & Seizures & Vigil & $\begin{array}{l}\text { Upper digestive } \\
\text { endoscopy (-) }\end{array}$ & \\
\hline & & Hypertension & Todd's paresis & Attentive & ECG (-) & Blood Transfusion \\
\hline & & Smoking Suspended & & Todd's phenomenon & ECHOC (-) & Asymptomatic \\
\hline & & $\begin{array}{l}\text { Pa ro xystic Atrial } \\
\text { Fibrillation }\end{array}$ & & & EEG (-) & \\
\hline & & $\begin{array}{l}\text { Recent Hospitalization } \\
\text { (Gastric ulcers) }\end{array}$ & & & Hto $20 \%$ & \\
\hline & & & & & $\mathrm{Hb} 7,5$ nigicll & \\
\hline & & & & & Platelets & \\
\hline & & & & & $782.000 \mathrm{mg} 111$. & \\
\hline & & & & & $1 \mathrm{Nlt} 1.29$ & \\
\hline \multirow[t]{4}{*}{3} & 64 - Female & Hypertension & $\begin{array}{l}\text { Consciousness } \\
\text { disturbance }\end{array}$ & Agitation & $\begin{array}{l}\text { EEG: encephalopathy } \\
\text { pattern }\end{array}$ & $\begin{array}{l}\text { Good response to } \\
\text { benzodiazepines and } \\
\text { quetiapine }\end{array}$ \\
\hline & & Smoking & $\begin{array}{l}\text { Psychomotor } \\
\text { Agitation }\end{array}$ & Inattentive & ECG (-) & Neurorehabilitation \\
\hline & & & & Left Hemiplegia & ECHOC (-) & \\
\hline & & & & & LP (-) & \\
\hline
\end{tabular}

HD, hemodialysis; ECG, electrocardiogram; ECHOC, echocardiogram; LP, lumbar punction; EEG, electroencephalogram; (-), None pathologic event

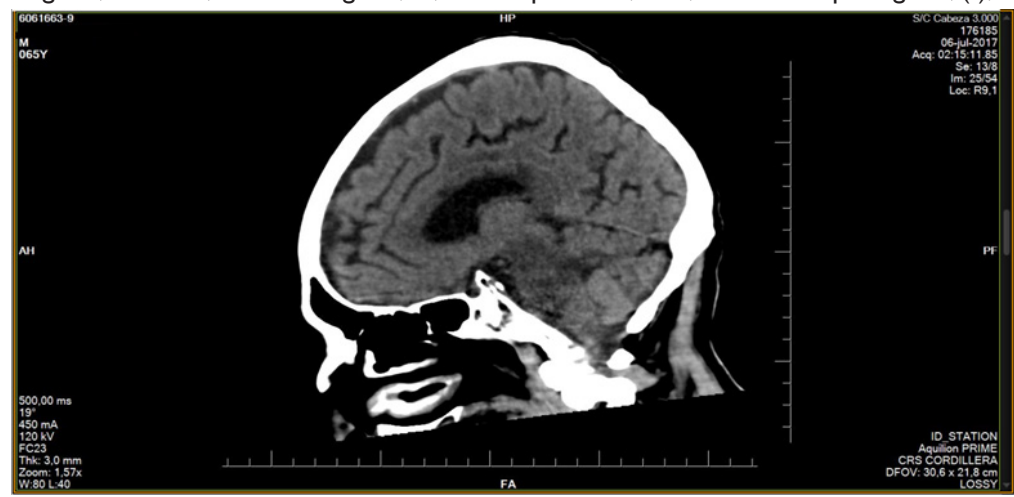

Figure I Sagittal image showing hypodense lesion in the splenium callosum corpus.

Citation: Cañas AV,Torrealba RG,Villar NR. Clinical spectrum of callosum corpus splenium lesions: subdiagnostics of a common entity.J Neurol Stroke. 2018;8(2):74-78. DOI: 10.15406/jnsk.2018.08.00283 

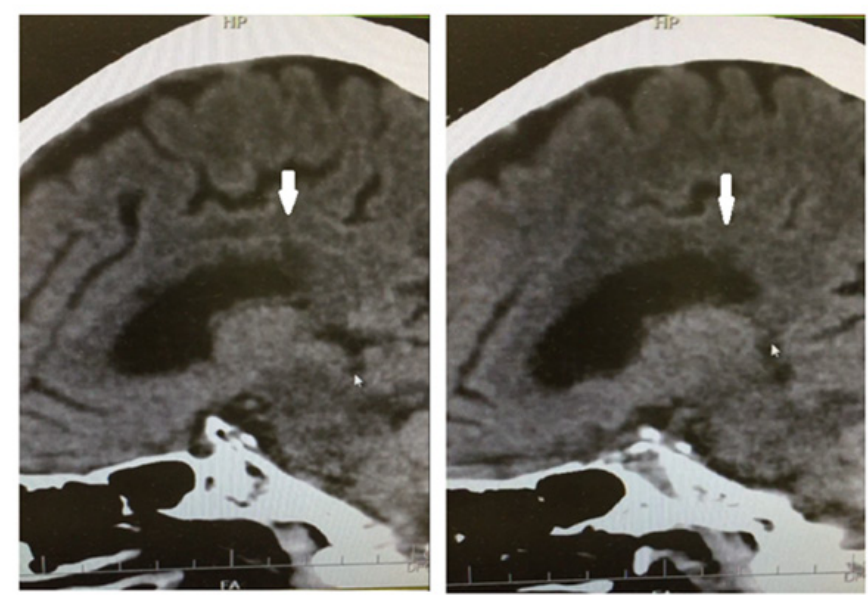

Figure 2 Sagittal image showing hypodense lesion in the splenium callosum corpus.

\section{Case 3}

A 64-year-old female; with hypertension and smoking habit of 15 cigarettes per day; did consultation to the ED by a disturbance of consciousness of 24 to 36 hours with severe psychomotor agitation. The first ED evaluation described a vigil patient, with dysarthria, delusional ideas and left hemiplegia. Is evaluated by psychiatry in ED, whom request $\mathrm{CT}$ without acute lesion and serum laboratory that show hematocrit in $29 \%$, red blood cell count in 3'200'000, electrolytes and creatinkinasa normal; but creatinine in 6 and BUN in 56. A diagnosis of acute over chronic renal disease is made. In ED, patient requires high doses of benzodiazepines for agitation, by this reason, is referral to intensive care unit, where it is sedated with propofol. It evolves with difficulty to arousal, with incoherent language, and delusional ideas. A treatment whith Quetiapine is initiates at dose of $300 \mathrm{mg}$ per day, with reversion of the symptoms. A LP was made, with cerebrospinal fluid (CSF) normal; and a EEG was performed, that shows encephalopatic pattern. Is studied again with cerebral tomography, which shows hypodense lesion in splenius of the corpus callosum (Figure 3) (Table1). The patient is transferred to the NU, whit clinical improvement; and in a posterior cerebral tomography the lesion was not present.

\section{Discussion}

Previous studies, ${ }^{1-3}$ classify the splenial callosum lesions topographically in isolated and multiple lesions. The first of them are most frequent and with best prognosis. Because the clinic is non-specific, and the etiology is unclear; it is expected that the pathophysiological mechanism of this type of lesion are not elucidated yet. There are many hypotheses; like intramielinic edema, axonal damage, hyponatremia, hyperglycemia, oxidative stress, malnutrition among others. ${ }^{1,3-7}$
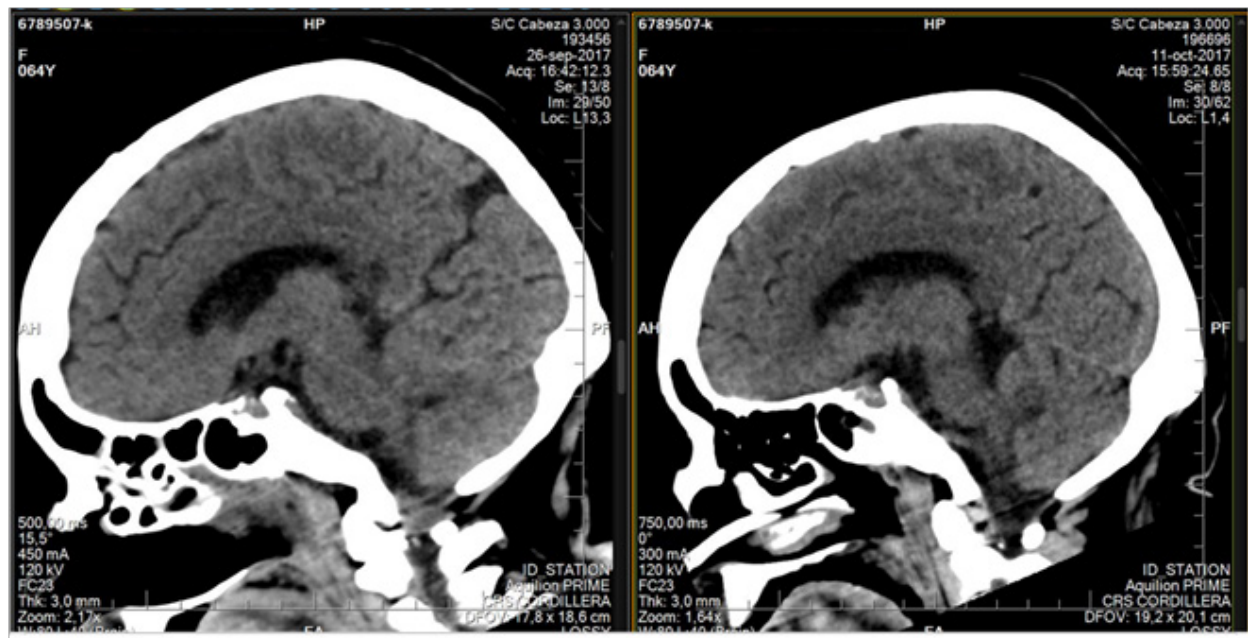

Figure 3 (A) Sagittal image showing hypodense lesion in the splenium callosum corpus. (B) Same patient after clinical recovery.

Two of our cases have pathophysiological mechanism was not clear, like other previous authors described in the literature, mainly because both patients had important comorbidities; for example, case 01; poor diabetic metabolic control and subtherapeutic treatment of hypertension; and case 02 ; recent upper gastrointestinal bleeding with severe iron deficiency anemia. By this reasons, is that we postulate that the damage's mechanism is multifactorial, as was mentioned by Polster. ${ }^{6}$ In individuals in whom it was associated with a seizure; like case number 02, LHECC can occur secondary to focal edema as a result of an alteration of BBB through elevation of capillary hydrostatic pressure. ${ }^{1,7}$ Here, we cannot rule out a transient ischemic attack, considering atrial fibrillation and severe anemic syndrome, so the LHECC could be just a finding, such as Pekala said, ${ }^{4}$ also considering that there was not disturbance of consciousness describe at all.

In cases where there is an associated systemic infection; it's postulated that the pathophysiological mechanism would to be a myelinic axonal damage, produced either by the antigen itself, or due the antibodies reaction against the antigen that alters the permeability of the BBB generating a focal edema. ${ }^{1,3}$ Considering that our case patient 01 was infected, this could have been one of the mechanisms that can caused the hypodense in the CC, as suggest Yuan et al. ${ }^{3}$

Also, the literature mentions some cases attributed to antiepileptic drug toxicity. ${ }^{1,7}{ }^{17}$ In consequence the manifestations of LHECC are variable; and is difficult to evaluate because the large number of comorbidities. Also these type of lesions can be found in asymptomatic individuals. Polster, ${ }^{6}$ found these lesions in 3 asymptomatic individuals, who were to undergo neurosurgical processes due to seizures refractory to medical treatment. Pekala, ${ }^{4}$ found these 
lesions in asymptomatic, elderly individuals with no relevant morbid antecedents. Clinical cases 1 and 3, both lesions were described in the evaluation of a confusional syndrome; while case 2 in a focal seizure, followed by self-limited abrupt left hemiparesis. In both patient: 2 and 3; there were found focal neurologic deficit at the ED evaluation (Figure 3).

It is necessary to clarify that the lesions in the first instance were not identifiable in any of our cases; probably due to the poor image resolution that the CT vs MRi used in the majority of previous reportsand despite this considerations; other authors agree on the difficulty of evaluating the splenius lesion of the corpus callosum even using MRI. ${ }^{1-4,6-10}$

Another conditions in our patients; was the many comorbidities associated, with a neurological examination without neurological focus that would to delay the diagnosis. In the cases 01 and 03 , there was an acute renal disease, and the needs of hemodialysis in one of them; this finding, could presume if this type of lesions would be secondary to uremia. This is a question that would require more studies of series of patients with chronic kidney disease, hemodialysis and uremic syndrome; but has been previously reported in patients with hemolytic-ureic syndrome. ${ }^{11,12}$ In none of our patients, a chronic dialisys was indicated.

We propose that a LHECC has a clinical relevance when is concomitant to a confusional syndrome or disturbance of consciousness, as Min-Keun Park ${ }^{2}$ and Junliang ${ }^{3}$ and Juan postulate in their respective publications; ${ }^{2,3}$ and was evident in cases 01 and 03.
On the other hand; like case 02 show, the clinically presentation of Todd phenomenon without unconsciousness forced to do differential diagnosis with a transient ischemic attack, especially in the context of a probably hypoperfusion with severe active anemia, thrombocytosis reactive or non-anticoagulated paroxystic atrial fibrillation as our patient had.

Due to the increasingly frequent neuroimaging of higher and better resolution, this type of lesions is expecte to be identified more frequently. Previous studies ${ }^{2,4,9}$ show that these lesions are more frequent than expected. Chrysikopoulos ${ }^{8}$ performed a retrospective study of 352 patients with neuroimaging; and did found 28 cases with lesions in the corpus callosum, in greater proportion in the splenium. Pekala, ${ }^{4}$ nevertheless questions why the splenium callosum lesions are being reported in more frequency nowadays and not before despite the neuroimaging are available years ago?

Finally; we also mentioned the importance of evaluating the images not only in axial plane, but also sagittal to be able to show structures and lesions that are easily altered by artefacts or simply not evidence at the axial one. Because our NU doesn't have MRI, we affirm due the high frequency found in consecutive months, that the splenius lesions of the corpus callosum are more common than expected, and should be a good practice try find them patients with the characteristics described above and reported in the literature; and keep in mind the differential diagnoses of corpus callosum lesions evidenced in CT (Table 2). These can be of variable etiology and have different presentations. ${ }^{13}$

Table 2 Description of Lesions involving the corpus callosum at CT

\begin{tabular}{|c|c|}
\hline $\begin{array}{l}\text { Glioblastoma } \\
\text { Multiforme }\end{array}$ & $\begin{array}{l}\text { Irregular, heterogeneous contrast enhancement, mass effect, vasogenic edema. Internal areas of necrosis, calcification } \\
\text { and hyperdense of hemorrhage. }\end{array}$ \\
\hline Lymphoma & $\begin{array}{l}\text { Rare, immunocompromised. Increased density, multifocal and nodular lesions with mass effect and peritumoral } \\
\text { vasogenic edema. Regresses rapidly with steroids "vanishing lesions" }\end{array}$ \\
\hline Meningioma & Increased density with internal calcifications \\
\hline Metastasis & Rare. Mass effect, vasogenic edema, heterogeneous contrast enhancement \\
\hline Multiple Sclerosis & Has a very low positive predictive value \\
\hline Head Trauma & Frequently involved. Can shows hyperdense petechial foci of hemorrhage \\
\hline $\begin{array}{l}\text { Hypoxemic Ischemic } \\
\text { Encephalopathy }\end{array}$ & Multiple hypodense lesions. \\
\hline $\begin{array}{l}\text { Marchiafava - } \\
\text { Bigmani }\end{array}$ & $\begin{array}{l}\text { Severe vitamin B12 deficiency. Alcoholics. Hypodense lesion resulting in a "sandwich like appearance. Minimal contrast } \\
\text { enhancement }\end{array}$ \\
\hline $\begin{array}{l}\text { Arteriosvenous } \\
\text { Malformation }\end{array}$ & $\begin{array}{l}\text { May affects both hemispheres. Identifying callosal hemorrhage, edema, mass effect and also can help delineate the } \\
\text { feeding arteries and draining veins }\end{array}$ \\
\hline
\end{tabular}

\section{Conclusion}

The LHECC, has multifactorial etiologies, without a clear mechanism, but usually, they are presented in relation to important comorbidities of the patients, mainly chronic non- transmissible diseases with poor metabolic control; and could be secondary to a uremic syndrome. Not all lesions in the splenius of the corpus callosum are clinically significant; so it is important to correlate them with the symptomatology; especially if there are found in relation to consciousness disturbance and confusion; and in these cases, keep in mind to evaluate the sagittal sections of the cerebral neuroimaging. Finally, and as other reports conclude, we also propose that these lesions are much more frequent than previously described in the literature.

\section{Acknowledgements}

To our patients, their trust is our golden rule.

To the authorities of our hospital, who supported our initiative.

To our families who always believe in us.

\section{Conflict of interest}

The authors declare not to have any financial interest or any conflict of interest in the realization of this publication. 


\section{Patient consent form}

All patients signed an informed consent form to authorize the use of the dates in this publication, assuring them the confidentiality of their identities.

\section{References}

1. Hardeep S, Ravindra K, Mukund R, et al. Boomerang sing: Clinical significance of transiet lesion in splenium of corpus callosum. Ann Indian Academy of Neurology. 2012;15(2):151-157.

2. Min-Keun Park, Sung-Hee Hwang, San Jung, et al. Lesions in the splenium of the corpus callosum: Clinical and radiological implications. Neurology Asia. 2014;19(1):74-88.

3. Junliang Yuan, Shuna Yang, Shuangkun Wang, et al. Mild encephalitis/ encephalopathy with reversible splenial lesion (MERS) in adults-a case report and literature review. BMC Neurology. 2017;17(1):103.

4. Pekala JS, Mamourian AC, Wishart HA, et al. Focal Lesion in the Splenium of the Corpus Callosum on FLAIR MR Images: A Common Finding with Aging and after Brain Radiation Therapy. AJNR Am J Neuroradiol. 2003;24(5):855-861.

5. Vargas A, Rivas M, Guerrero R, et al. Marchiafava-Bignami's Disease, as Etiologic Diagnosis of Athetosis. Ann Neurosc. 2017;24(1):57-60.

6. T Polster, M Hoppe, A Ebner. BMC Neurology. Transient lesion in the splenium of the corpus callosum: three further cases in epileptic patients and a pathophysiological hypothesis. J Neurol Neurosurg Psychiatry. 2001;70(4):459-463.
7. Hayat Guven, Sennur Delibas, Selim Selcuk Comoglu. Transient Lesion in the Splenium of the Corpus Callosum Due to Carbamazepine. Turk Neurosurg. 2008;18(3):264-270.

8. Chrysikopoulos H, Andreou J, Roussakis A, et al. Infarction of the corpus callosum: computed tomography and magnetic resonance imaging. Eur J Radiol. 1997;25(1):2-8.

9. Marvid Sol Duarte Moreno, Edna Julieth Bobadilla Quesada, Pablo Vásquez Hoyos, et al. Lesión esplénica reversible con encefalopatía/encefalitis leve (MERS). Acta Neurológica Colombiana 2016;32(4):325-9.

10. L Valls Masot, G Blasco Solà, J Puig Alcántara, et al. Lesiones del cuerpo calloso: diagnóstico diferencial, mediante técnicas convencionales y avanzadas de resonancia magnética". Sociedad Española de Radiología Medica. 31 ${ }^{\text {a }}$ Congreso nacional, Granada, España. 2012.

11. Ogura H, Takaoka M, Kishi M, et al. Reversible MR findings of hemolityc uremic syndrome with mild encepalopahy. Am J Neuroradiol. 1998;19(6):1144-1145.

12. Gawlitza M, Hoffmann KT, Losbien D. Mild encephalitis/ encephalopathy with reversible splenial and cerebellar lesion (MERS type II) in a patient with hemolytic uremic syndrome. J Neuroimaging. 2015;25(1):145-146.

13. Mai-Lan Ho, Gul Moonis, Daniel T Ginat, et al. Lesion of the corpus callosum. AJR 2013;200:W1-W6. 